\title{
A New Makespan Estimation Model for Scientific Workflows on Heterogeneous Processing Systems
}

\author{
D. Sirisha \\ Department of Computer Science and \\ Engineering, Pragati Engineering College, \\ Surampalem, India
}

\author{
G. Vijayakumari \\ Department of Computer Science and \\ Engineering, JNTUH College of Engineering, \\ JNTU, Hyderabad, India
}

\begin{abstract}
Scientific workflows epitomizing computation-intensive applications demand heterogeneous processing resources for attaining high performance. Generally, optimal scheduling of the tasks in workflow is well-acknowledged NP-complete problem. In the present work, a new makespan estimation model is proposed to estimate the bounds on the makespan of the workflows using minimal information. The performance of the proposed estimation model is evaluated using four scientific workflows and the estimation of the makespan computed by the model is compared with the actual makespan generated by the most-cited heuristic scheduling algorithms devised for heterogeneous processing systems. The experimental results revealed that the proposed estimation model is effective and can precisely estimate the makespan of the workflows with an error of over $10 \%$ and $26 \%$ for computation-intensive and data-intensive workflows respectively.
\end{abstract}

\section{General Terms}

High Performance Computing, Workflow Scheduling, makespan estimation

\section{Keywords}

scientific workflows; high performance; heterogeneous processing resource; makespan; makespan estimation

\section{INTRODUCTION}

Large monolithic applications in numerous scientific fields such as knowledge discovery, bioinformatics, computations, weather and climate modeling, earthquake science, genome analysis and astronomy can be broken down into smaller tasks structured with intricate dataflow dependencies among themselves are often structured as scientific workflows. Scientific workflows expedite scientists to nimbly model the computation intensive applications as multi-stage computational tasks usually involving a series of data processing operations such as data retrieving, transformation, analysis and aggregation stages. The complexity and the heterogeneity of the scientific workflows demands Heterogeneous Processing Resources (HPR) to attain high performance. HPR are the most potential platforms for rendering high performance at lower costs.

The contributions of the current work are

- A new makespan estimation model is proposed to estimate the bounds on the completion time, i.e., makespan of the scientific workflows.

- The proposed model estimates the Best-Case Computation Time (BCCT) and Worst-Case Computation Time (WCCT) of the scientific workflows using minimal information.
- The computational complexity of the new makespan estimation model is lesser than the existing models.

- Validation of the model is performed with the most-cited heuristic scheduling algorithms developed for HPR.

In general, workflow scheduling problem is a wellacknowledged NP-Complete problem [1]. However, the available estimation models deal with simple workflows having unit tasks and no dataflow latencies. Hence, the existing models are not viable for scientific workflows. The proposed estimation model is devised using minimal information, i.e., the profile of the scientific workflows. Generally, the profile of the scientific workflows constitutes of the height and the parallelism of the workflow. As the makespan of the workflow highly depends upon the profile of the workflows, these two parameters chiefly contribute for devising good and precise makespan estimation model which can satisfy all the cases.

A makespan estimation model is valid only when BCCT and WCCT functions are admissible. The admissibility of the estimating functions must guarantee that the BCCT never overestimates and WCCT never underestimates the actual makespan of the scientific workflow. Therefore, the estimation model must provide tight bounds on the makespan. The primary aim of the new makespan estimation model is to provide a-priori information of the bounds on the makespan i.e., BCCT and WCCT of the scientific workflows. The apriori information provided by BCCT and WCCT bound is required to schedule the workflows, provide the resources, and guides in devising the scheduling algorithms.

The remaining paper is structured as follows. In section II, the problem is described, and the overview of the scientific workflows is illustrated in section III. In section IV, the related work is detailed and in section $\mathrm{V}$, the new makespan estimation model is presented. In section VI, the proposed model is evaluated using four scientific workflows and finally section VII summarizes the present work.

\section{PROBLEM DESCRIPTION}

\subsection{Application Model}

A workflow $\mathrm{W}$ encompasses of a set of tasks which are connected in the form of a Directed Acyclic Graph, W $=<\mathrm{T}$, $\mathrm{E}\rangle$, where $\mathrm{T}$ is a set of $n$ tasks $\left\langle t_{1}, t_{2} \ldots t_{n}\right\rangle$, and $\mathrm{E}$ indicates a set of directed edges $\left\langle e_{i, j}, . . e_{m, n}\right\rangle$ forming no cycles. Each edge $e_{i, j}$ imposes dependency constraint between the tasks $t_{i}$ and $t_{j}$, i.e., the task $t_{j}$ can be executed only when its predecessor task $t_{i}$ is completed. Each task $t_{i} \in \mathrm{T}$ is associated with a positive integer $w_{i}$ which specifies its computation time. Moreover, each edge $e_{i, j} \in \mathrm{E}$ is associated with a non- 
negative integer $d_{i, j}$ that denotes the dataflow time between the tasks $t_{i}$ and $t_{j}$. A task having no

Table 1. Computation Time Matrix

\begin{tabular}{|c|c|c|}
\hline Task & \multicolumn{1}{|c|}{$\boldsymbol{r}_{\boldsymbol{1}}$} & $\boldsymbol{r}_{\mathbf{2}}$ \\
\hline$t_{0}$ & 70 & 84 \\
\hline$t_{1}$ & 68 & 49 \\
\hline$t_{2}$ & 78 & 96 \\
\hline$t_{3}$ & 89 & 26 \\
\hline$t_{4}$ & 30 & 88 \\
\hline$t_{5}$ & 66 & 86 \\
\hline$t_{6}$ & 25 & 21 \\
\hline$t_{7}$ & 96 & 26 \\
\hline
\end{tabular}

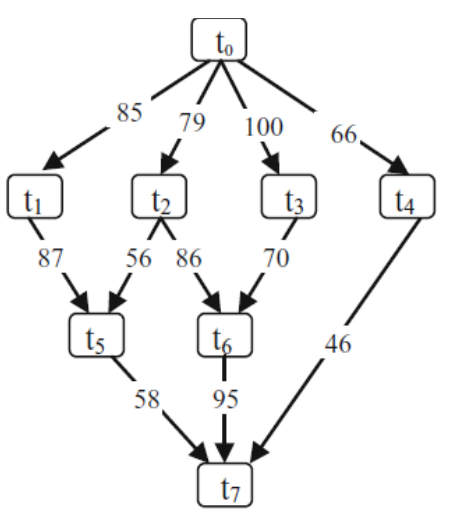

Fig 1: An example workflow

predecessor is termed as the start task $t_{\text {start }}$ and the task with no successor is the sink task $t_{\text {sink }}$. Generally, a workflow includes a start task and a sink task otherwise a pair of pseudo start, and sink tasks must be connected with pseudo edges to the numerous start and sink tasks. The matrix $\mathrm{D}$ of order $n \times n$ is used to represent the dataflow time between the tasks, where each element $d_{i, j}$ in the matrix $\mathrm{D}$ indicates the dataflow time between the tasks $t_{i}$ and $t_{j}$.

The organization of the tasks in a workflow describes the structure of the workflow. A task happens to be free upon the completion of all its predecessors and after it receives the data from its predecessors it becomes ready. As each task becomes ready it is placed in the queue for execution. An example workflow is shown in Figure 1.

\subsection{HPR Model}

A HPR model constitutes of a suite of $m$ resources $<r_{1}, r_{2} \ldots r_{m}$ $>\in \mathrm{R}$ with varied processing potentials, fully connected with high speed network. Each resource can execute only one task at a time i.e., tasks cannot be preempted. The execution of task $t_{j}$ and its predecessor $t_{i}$ on the same resource, i.e., $r\left(t_{i}\right)=$ $r\left(t_{j}\right)$, then the dataflow between the two tasks is assumed as local and dataflow time $d_{i, j}$ is zeroed. Since, the output data generated by the task $t_{i}$ is available at the same resource on which task $t_{j}$ is to be performed, transferring of the data is not required. Otherwise, i.e., $r\left(t_{i}\right) \neq r\left(t_{j}\right)$, the dataflow is assumed to be remote. Moreover, the computations of the tasks and the dataflow between the tasks are carried out simultaneously. The matrix $\mathrm{E}$ of order $n \times m$ is used to represent the computation time of $n$ tasks on $m$ resources and each element $w_{i, j}$ denotes the computation time of the task $t_{i}$ on resource $r_{j}$. Table. 1 shows the computation time matrix for the workflow depicted in Figure 1.

The key attributes required to describe the workflow scheduling are the Earliest Start Time (EST) and the Earliest Completion Time (ECT). The EST and ECT of the task $t_{i}$ on resource $r_{j}$ are denoted as $\operatorname{EST}\left(t_{i}, r_{j}\right)$ and $E C T\left(t_{i}, r_{j}\right)$ respectively and computed by (1) and (2) respectively. The EST for a start task $t_{\text {start }}$ is zero.

$$
\begin{gathered}
\operatorname{EST}\left(t_{i}, r_{j}\right)=\max \left\{\operatorname{ready}\left(t_{i}\right), \operatorname{avail}\left(r_{j}\right)\right\} \\
E C T\left(t_{i}, r_{j}\right)=\operatorname{EST}\left(t_{i}, r_{j}\right)+w_{i, j}
\end{gathered}
$$

where $\operatorname{ready}\left(t_{i}\right)$ is the time the task $t_{i}$ becomes ready and avail $\left(r_{j}\right)$ is the time the resource $r_{j}$ is available to execute the next task. In the equation (2), ECT( $\left.t_{i}, r_{j}\right)$ is computed by $\operatorname{EST}\left(t_{i}, r_{j}\right)$ and $w_{i, j}$, where $w_{i, j}$ is the computation time of the task $t_{i}$ on resource $r_{j}$. The schedule length, i.e., makespan of $\mathrm{W}$ is the Actual Completion Time (ACT) of $t_{s i n k}$, given by (3)

$$
\text { makespan }=A C T\left(t_{\text {sink }}\right)
$$

Definition 1 (Bottom Level). The bottom level of task $t_{i}$ is the length of the longest path from the task $t_{i}$ to $t_{\text {sink }}$. It is denoted as $b l\left(t_{i}\right)$ and computed by the computation and dataflow times along the path using (4)

$$
b l\left(t_{i}\right)=\overline{w_{i}}+\max _{t_{j} \in \operatorname{succ}\left(t_{i}\right)}\left\{b l\left(t_{j}\right)+d_{i, j}\right\}
$$

where $\overline{w_{i}}$ is the average computation time of the task $t_{i}$ and $\operatorname{succ}\left(t_{i}\right)$ is a set of immediate successors of $t_{i}$ and $d_{i, j}$ is the dataflow times between the tasks $t_{i}$ and $t_{j}$.

Definition 2 (Top Level). The top level of the task $t_{i}$ is the length of the longest path from the start task $t_{\text {start }}$ to $t_{i}$, excluding the computation time of $t_{i}$ It is denoted as $t l\left(t_{i}\right)$ and computed by the computation and dataflow times along the path using (5)

$$
\operatorname{tl}\left(t_{i}\right)=\max _{t_{j} \in \operatorname{pred}\left(t_{i}\right)}\left\{t l\left(t_{j}\right)+w_{j}+d_{j, i}\right\}
$$

where $\operatorname{pred}\left(t_{i}\right)$ is a set of immediate predecessors of $t_{i}$ and $d_{j, i}$ is already explained in the equation (4).

Definition 3: The Critical Path (CP) is the longest path in the workflow and the length of the $\mathrm{CP}$ can be computed by the sum of the computation and dataflow times along the path and denoted as $C P_{l}$. The computation critical path is computed by the sum of the minimum computation time of each task on $\mathrm{CP}$, it is denoted as $C P_{c}$ and computed using (6).

$$
C P_{c}=\sum_{t_{i} \in \mathrm{CP}} \min _{r \in \mathrm{R}} w_{i}
$$

The CP tasks in a workflow can be identified by summing up $b l\left(t_{i}\right)$ and $t l\left(t_{i}\right)$ for each task $t_{i}$. All the tasks on CP have same $\left(b l\left(t_{i}\right)+t l\left(t_{i}\right)\right)$ value which is equal to $C P_{l}$.

\section{THE RELATED WORK}

Limited research work has been carried out in estimating the performance of the workflows.

Fernandez et al. [4] were the first to devise lower bounds on the makespan for the workflows with unit sized tasks having no dataflow latencies and performed on homogeneous environments. To compute the lower bound, an interval $\left[\theta_{1}, \theta_{2}\right]$ during which maximum number of tasks $n^{\prime} \subset n$ can be executed is considered such that $\left[\theta_{l}, \theta_{2}\right] \subset\left[0, C P_{c}\right]$. For $m$ resources, and $n$ tasks in the workflow, then $m \times\left(\theta_{1}-\theta_{2}\right)$ 
denotes the tasks executed on $m$ resources. The excess area is divided by $m$ and added to $C P_{c}$.

The WCCT, i.e., the upper bound of the workflow was first proposed by Jain et al. [5]. The computation of the upper bound was confined to the workflows consisting of unit computation time tasks having no dataflow overheads and was executed on homogeneous systems. The workflow is partitioned according to the levels and the upper bound for each partition was computed by the summation of the number of ready tasks divided by $m$. Finally, the upper bounds of all

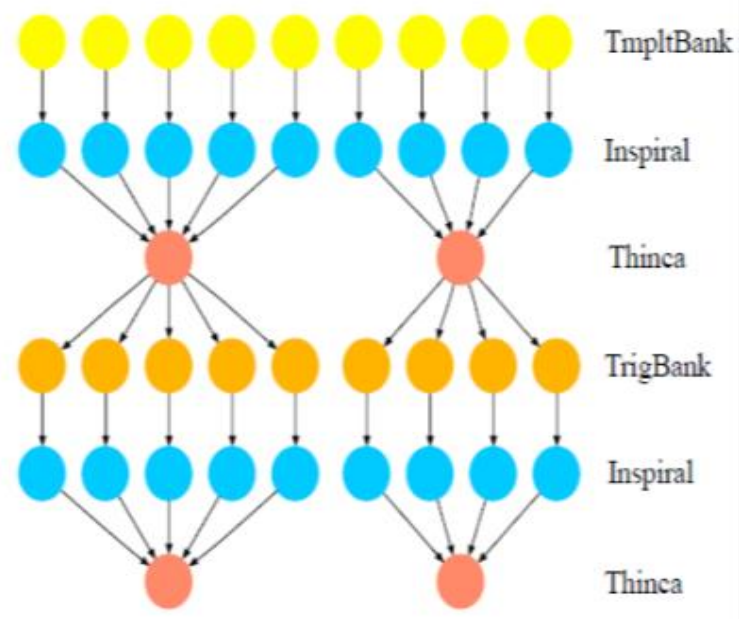

(a) LIGO

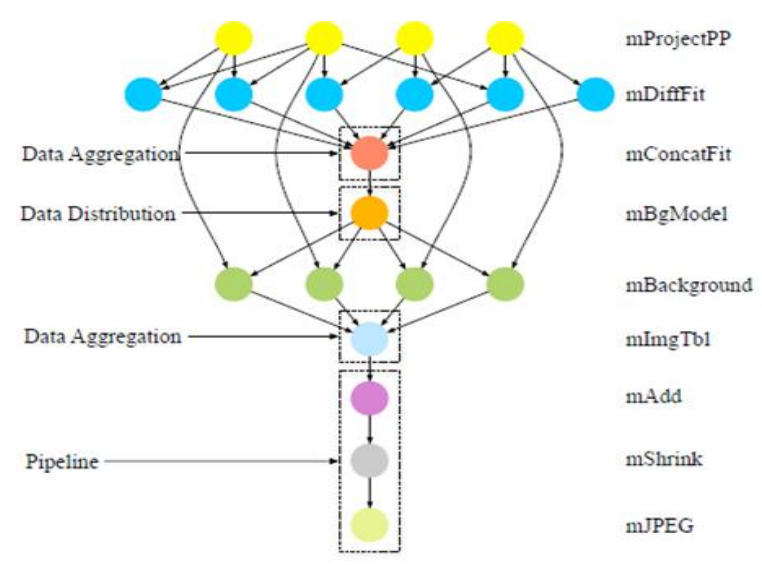

(c) Montage the partitions are summed up to obtain the upper bound of the workflow. Moreover, the lower bound is computed by considering a partition in which the maximum number of tasks can be executed and is divided by $m$.

In [8], a level based estimation model is proposed to analyze the computation times of the workflows. The model requires the workflow to be partitioned into levels in both top-down and bottom-up approaches to group the set of tasks which can be performed concurrently. The level computation time is computed by the sum of the computation times of all the tasks

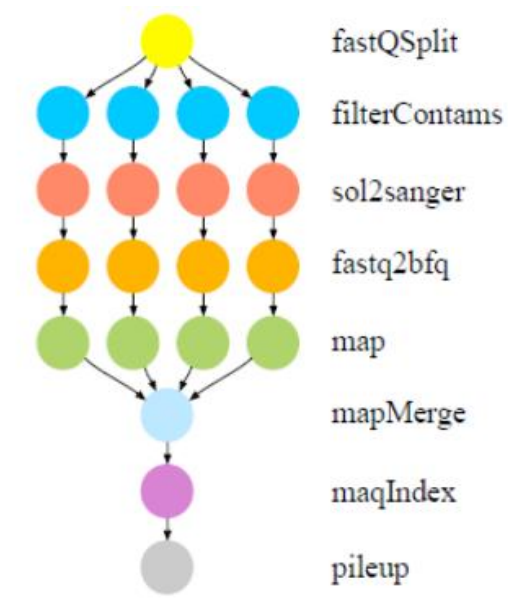

(b) Epigenomics

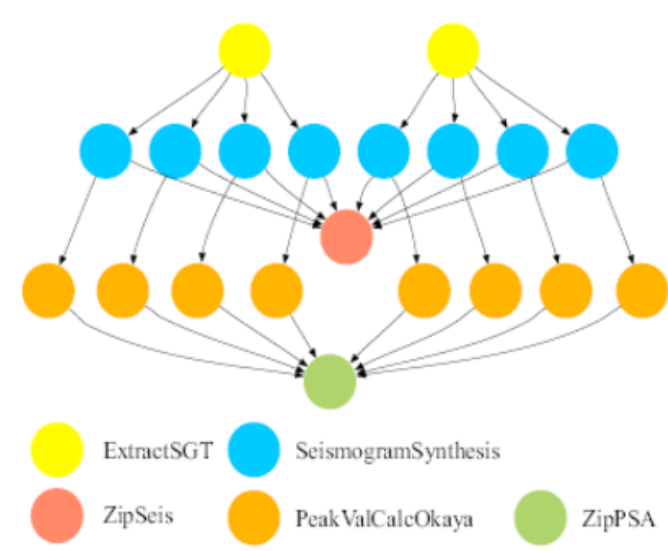

(d) Cybershake

Fig 2: The structure of the scientific workflows

in a level. The makespan for each level is computed by the maximum of the level computation time divided by $m$ and the maximum computation time of a task at that level. The performance of the workflow is estimated by the summation of the makespan of all the levels and nominal dataflow latency is added to it.

\section{AN OVERVIEW OF SCIENTIFIC WORKFLOWS}

Generally, scientific applications are categorized as computation-intensive, data-intensive, memory -intensive, or a combination of these based upon the applications. The computation-intensive workflows consist of tasks which expend most of the time in computations, while the tasks in the data-intensive workflows generate enormous data and therefore involve much in exchanging data rather than computations [6]. The tasks in the memory-intensive workflows demand high physical storage requirements. Most of the scientific applications embrace workflows to epitomize computation- intensive applications for efficient computation on heterogeneous environments which are the most preferred platforms for attaining high performance.

Laser Interferometer Gravitational Wave Observatory (LIGO) is an Inspiral Analysis Workflow [11]. LIGO is a memory bound and heavily computation-intensive workflow that detects gravitational waves generated by numerous events in the universe according to Einstein's theory of relativity. The LIGO workflow structure allows greater parallelism. This workflow is applied to analyze the data acquired by 
amalgamating of compact binary systems, namely binary neutron stars and black holes. The time-frequency data from any event for each of the three LIGO detectors is fragmented into small chunks and then analyzed. A set of waveforms which belong to the parameter space are generated for each chunk. Matched filter output is computed and triggers if Inspiral is detected which are then tested for consistency by the Thinca jobs. Inspiral jobs are highly computationintensive. Trigger output generates Template banks. The structure of the LIGO workflow is depicted in Figure 2(a).

Epigenomics [12] is a computation-intensive and largely data parallel pipelined application. This workflow primarily processes pipelined data to execute genome sequence jobs which is utilized by Maq system. The remaining jobs of the workflow include filter of noise and contaminated data, a map job which aligns the current location in a reference genome, which is mostly computation-intensive and generates global map to identify the sequence density at each position in the genome. A simple way to enhance performance is to run Epigenomics on heterogeneous platforms since many tasks of this application can run in parallel and are heterogeneous and the structure of this application is given in Figure 2(b).

Montage workflow is devised by NASA / IPAC [9] is an astronomical image mosaic engine which is more widely studied workflow applications. Montage is designed to take multiple astronomical images from telescopes or any other instruments and amalgamate into a single mosaic that appears to be taken from a single instrument. The input images are reprojected onto a sphere and the overlap of the images is calculated. Subsequently, the images are re-projected to correct the orientation and to normalize the background. Lastly, all the processed images are composed into a single mosaic. Astronomers can tailor the functionalities of Montage workflow and add code according to the requisites. Accordingly, Montage is designed consisting of a set of tasks and mostly, the tasks spend much time in communicating and very lesser amount of time in the computation and hence Montage is described as data-intensive workflow and its structure is shown in Figure 2(c).

Cybershake is a seismological application which is highly data and memory-intensive and its structure is depicted in Figure 2(d). This application is generated by the Southern California Earthquake Center [10] to characterize earthquake hazards in geographical regions using Probabilistic Seismic Hazard Analysis (PSHA) technique. The application utilizes Earthquake Rupture Forecast (ERF) to detect the probable ruptures within $200 \mathrm{~km}$ of an interested region. For every rupture, rupture definitions are converted from ERF into numerous rupture variations with varying hypocenter positions and slip time distributions to generate Strain Green Tensors (SGT). Subsequently, synthetic seismograms for every rupture variation are computed and peak intensity measures are derived from the synthetics which are then combined with the original rupture probabilities to generate probabilistic seismic hazard curve in the region. The information provided by PSHA is utilized by city planners and building engineers to estimate seismic hazards prior to construction of the buildings.

\section{A NEW MAKESPAN ESTIMATION MODEL}

Basically, the makespan of the workflow is influenced by the profile of the workflow, dataflow latencies between the tasks in the workflow and the number of resources employed in the computation of the workflow.
The profile of the workflow includes the implicit parallelism and the height of the workflow. The execution of the parallel tasks concurrently on an adequate number of resources can significantly reduce the makespan. On the other hand, the height of the workflow reflects the longest path in the workflow, i.e., the CP. Because of the sequential bottlenecks of the CP tasks, these tasks can only be performed serially and hence provisioning of additional resources may not further minimize the makespan.

The main shortcomings of the existing models in the literature are they do not consider the dataflow latencies among the tasks and hence do not fit for the scientific workflows. On the other hand, the new makespan estimation model is devised considering the Critical Path (CP), and the intrinsic parallelism of the workflow. The proposed estimation model captures the workflow parallelism by effectively identifying the independent branches in a workflow. CP is a global heuristic and the inherent sequential path of the workflow that plays a pivotal role in determining the bounds on the computation time.

Theorem 1: Let $W=\langle T, E, w, d\rangle$ be a workflow and $C P_{c}$ be the compute critical path. For any schedule $S$ of $W$, the makespan is always greater than $C P_{c}$.

$$
\text { makespan }>\mathrm{CP}_{c}
$$

Proof: A CP always begins at the start task and ends at the sink task and it is the longest directed path between a pair of start and sink tasks. A directed path in the workflow represents the chain of dependent tasks that are to be processed in a sequence. Any other path length in the workflow is at least the length of the computation critical path length. Therefore, the duration of the workflow is at least $C P_{c}$ regardless of the number of resources provisioned.

Lemma 1: The maximum number of levels in a workflow in any path cannot exceed the number of tasks on the critical path.

Proof: Let a workflow W constitutes of $k$ number of levels, $\ell=\left\{\ell_{1}, \ell_{2}, \ell_{3}, \ldots \ell_{k}\right\}, 1 \leq k \leq n$. The tasks can be grouped into the levels by topologically ordering the tasks $t_{i} \in \mathrm{T}$ in $\mathrm{W} \ni$ for each edge $e_{i, j} \in \mathrm{E}$, if $t_{i}$ is in the level $\ell_{k-1}$ then $t_{j}$ must occur in the level $\ell_{k}$, where $k>j$. Therefore,

$$
\ell=\bigcup_{k=1}^{n} \ell_{k}=\mathrm{T}
$$

As per definition 1, CP is the longest path from the start task to sink task. At most one task from each level $\ell_{k}, 1 \leq k \leq n$, lies on $\mathrm{CP}$, i.e., no more than $k$ tasks can be included in $\mathrm{CP}$, which are denoted as $t_{c p, 1}, t_{c p, 2}, . . t_{c p, k}$, where each task $t_{c p, i}, 1 \leq i$ $\leq k$, indicates $i^{\text {th }}$ task on CP selected from level $\ell_{i}$. Moreover, there may be more than one $\mathrm{CP}$ in a workflow as several paths can have maximal length.

Lemma 2: The maximum queue length equals the level maximum branching factor of the workflow.

Proof: The maximum branching factor of the workflow determines the queue length. The maximum queue length is the maximum number of tasks that become free upon the execution of a task. For example, consider the workflow given in the Figure 1 scheduled on two resources. However, the execution of the task $t_{o}$ at the level 1 frees the tasks $t_{1}, t_{2}, t_{3}$, and $t_{4}$ at level 2 . Therefore, the maximum queue length for the given workflow is four. 
Lemma 3: When the ready tasks are more than the number of resources, then in an ideal scheduling system the load is equally balanced on all the resources. The maximum additional load on each resource is no more than the queue length $/ m, m$ is the number of resources on HPR.

Proof: Let $k \subseteq n$ be the number of ready tasks placed in the queue and $m$ be the number of resources on HPR to execute a workflow. During the computation of the workftow, if $k$ exceeds $m$, then $(k-m)$ tasks fall as additional load on $m$ resources. In an ideal scheduling system this additional load is equally distributed on $m$ resources. Therefore, the additional load on each resource is no more than queue length $/ \mathrm{m}$.

For instance, in the given workflow in the Figure 1, if the task $t_{o}$ at the level 1 at level 1 is executed, frees the tasks $t_{1}, t_{2}, t_{3}$, and $t_{4}$ which are added to queue. Since the workflow is executed on two resources, only two tasks can be executed in parallel. In an ideal scheduling system, the remaining two tasks are distributed to two resources which is the additional load incurred on each resource due to the inadequate resources provisioned for performing the workflow. Therefore, queиe length $/ \mathrm{m}$ is the additional load on each resource that eventually leads to the increase in the makespan.

Theorem 2: The WCCT of a workflow equals the $C P l+\varepsilon$, where $\varepsilon$ is the additional load taken by each resource when the number of resources in HPR is less than queue length.

$$
\text { makespan } \leq\left\{\begin{array}{cc}
C P_{l}, & k<m \\
C P_{l}+\varepsilon, & \text { otherwise }
\end{array}\right.
$$

Proof: Generally, the profile of the workflow depends upon two parameters, i.e., $\mathrm{CP}$ of the workflow and the number of parallel tasks. As per definition 1, CP is the longest chain of dependent tasks in the workflow and hence these tasks must be performed in succession. According to Lemma 1, the maximum number of levels in a workflow cannot be more than the number of tasks lying on CP. The length of any path in the workflow is always lesser than or equal to the length of $\mathrm{CP}$, i.e., $C P_{l}$. Consequently, the constrained sequential computation time of the tasks on the $\mathrm{CP}$ is at most the completion time of any path in the workflow. Therefore, the WCCT of a workflow is chiefly contributed by the critical path length with respect to the number of levels.

The second parameter $\varepsilon$ represents the number of tasks that can be performed in parallel provided when sufficient resources are provisioned to execute the workflow on HPR. In situations when fewer resources are provisioned, the queue length gradually increases and falls as an additional load on each resource and thus leads to the increase in the makespan. Therefore, $\varepsilon$ is the maximum additional load on all the resources, i.e., maximum of queue length $/ \mathrm{m}$.

In situations when the maximum branching factor $(k)$ of the workflow is greater than the number of resources $(m)$ provisioned to execute a workflow, i.e., $k>m$, the queue length gradually increases which falls as an additional load on each resource and leads to the increase in the makespan. According to Lemma 3, $\varepsilon$ is the maximum additional load on all the resources, i.e., maximum of queue length $/ \mathrm{m}$, computed as follows.

$$
\varepsilon=(k-m) * \bar{w} / m
$$

where $\bar{w}$ is the average execution time of each task in the workflow. The equation (9) establishes a sharp WCCT of the workflow. Therefore, BCCT and WCCT values are chiefly contributed by $\mathrm{CP}$ of the workflow and the completion time of a workflow is highly dependent on the structure of the workflow. Figure 3 and Figure 4 present the algorithm for WCCT and BCCT for the workflow.

Algorithm 1. A new makespan estimation model for WCCT of the workflow

Input: $\mathrm{A}$ workflow $\mathrm{W}=\left\langle\mathrm{T}, \mathrm{E}, w_{i}, d_{i, j}\right\rangle, \mathrm{T}$ and $\mathrm{E}$ are a set of tasks and edges in the workflow. $\overline{w_{i}}$, is the average computation time of the task $t_{i}$ and $d_{i, j}$ is the dataflow time between the tasks $t_{i}$ and $t_{j}$.

Output: The WCCT of the workflow.

1. Set the average computation time $\overline{w_{i}}$ for each task $t_{i} \in \mathrm{T}$.

2. Compute $b l$ for $t_{i} \in \mathrm{T}$ tasks in W.

3. Compute $t l$ for $t_{i} \in \mathrm{T}$ tasks in $\mathrm{W}$.

4. Compute $\operatorname{rank}\left(t_{i}\right)=b l\left(t_{i}\right)+t l\left(t_{i}\right)$ for $t_{i} \in \mathrm{T}$ tasks in $\mathrm{W}$.

5. Compute $C P_{l}:=\operatorname{rank}\left(t_{\text {start }}\right)$, where $t_{\text {start }}$ is the start task.

6. $C P_{\text {set }}:=t_{\text {start }} / / C P_{\text {set }}$ is the set of CP tasks.

7. $t_{i} \leftarrow t_{\text {start }}$

8. while $\left(t_{i} !=t_{\text {sink }}\right)$ do

9. Select $t_{j}$ where $\left(\left(t_{j} \in \operatorname{succ}\left(t_{i}\right)\right)\right.$ and $\left(\operatorname{rank}\left(t_{j}\right)==\right.$ $\left.\left.C P_{l}\right)\right)$. // $\operatorname{succ}\left(t_{i}\right)$ is the successor tasks of $t_{i}$

10. $C P_{\text {set }}:=C P_{\text {set }} \cup\left\{t_{j}\right\}$

11. $\operatorname{if}(k>m) / /$ where $k$ is the maximum branching factor of the workflow and $m$ is the number of resources provisioned to execute a workflow

12. $\varepsilon=(k-m) * \overline{w_{i}} / m \quad / /$ where $\varepsilon$ is the maximum additional load on all resources

13. WCCT $:=\mathrm{WCCT}+\overline{w_{i}}+\varepsilon$

14. else

15. WCCT $:=$ WCCT $+\overline{w_{i}}$

16. $t_{i} \leftarrow t_{j}$

17. end while

Fig 3: Algorithm for estimating WCCT of the workflow

Algorithm 2. A new makespan estimation model for BCCT of the workflow

Input: $\mathrm{A}$ workflow $\mathrm{W}=\left\langle\mathrm{T}, \mathrm{E}, w_{i}, d_{i, j}>, \mathrm{T}\right.$ and $\mathrm{E}$ are a set of tasks and edges in the workflow. $w_{i}$ is the minimum computation time of the task $t_{i}$ and $d_{i, j}$ is the dataflow time between the tasks $t_{i}$ and $t_{j}$.

Output: The BCCT of the workflow.

1. Set $w_{i}$ to the minimum computation time of each task $t_{i} \in$ $\mathrm{T}$.

2. Compute $b l$ for $t_{i} \in \mathrm{T}$ tasks in $\mathrm{W}$.

3. Compute $t l$ for $t_{i} \in \mathrm{T}$ tasks in $\mathrm{W}$.

4. Compute $\operatorname{rank}\left(t_{i}\right):=b l\left(t_{i}\right)+t l\left(t_{i}\right)$ for $t_{i} \in \mathrm{T}$ tasks in $\mathrm{W}$

5. $C P_{l}:=\operatorname{rank}\left(t_{\text {start }}\right)$, where $t_{\text {start }}$ is the start task.

6. $C P_{\text {set }}:=t_{\text {start }} / / C P_{\text {set }}$ is the set of $\mathrm{CP}$ tasks.

7. $t_{i} \leftarrow t_{\text {start }}$

8. while $\left(t_{i} !=t_{\text {sink }}\right)$ do

9. $\quad$ select $t_{j}$ where $\left(\left(t_{j} \in \operatorname{succ}\left(t_{i}\right)\right)\right.$ and $\left.\left(\operatorname{rank}\left(t_{j}\right)==C P_{l}\right)\right)$ $/ / \operatorname{succ}\left(t_{i}\right)$ is the successor of $t_{i}$

10. $\quad \mathrm{CP}_{\text {set }}:=\mathrm{CP}_{\text {set }} \cup\left\{t_{j}\right\}$

11. $\mathrm{BCCT}:=\mathrm{BCCT}+w_{j}$

12. end while.

Fig 4: Algorithm for estimating BCCT of the workflow 


\subsection{Time Complexity Analysis}

The computation of the WCCT of the workflow includes CP length, i.e., $C P_{l}$ and $\varepsilon$. The $\mathrm{CP}$ includes $n^{l}<n$ tasks and $e^{l}<e$ edges and hence $C P_{l}$ can be calculated in time $\mathrm{O}(n+e)$ while the computation of $\varepsilon$ involves $n^{l}<n$ tasks and requires $\mathrm{O}(n)$ time. Therefore, the complexity of WCCT of the workflow is $\mathrm{O}(n+e)$, where $n$ is the number of tasks and $e$ is the number of edges in the workflow. The computation of BCCT of the workflow entails $C P_{c}$. The calculation of $C P_{c}$ necessities $n^{l}<$ $n$ tasks and hence BCCT of a workflow can be computed in time $\mathrm{O}(n)$. Generally, the WCCT and BCCT of the workflow are computed recursively adding one task in each iteration.

The WCCT and BCCT values computed for the example workflow depicted in the Figure 1 are 508 and 195 respectively. The makespan for the same workflow generated by the HEFT [2], PETS [7] and MSL[3] scheduling strategies are 446, 446 383-time units respectively.

\section{PERFORMANCE EVALUATION}

In this section, the performance of the new makespan estimation model is evaluated using randomly generated scientific workflows namely LIGO, Epigenomics, Cybershake, and Montage. Generally, the characteristics required for generating the scientific workflows are as follows.

- Workflow size $(n)$ is the number of tasks in the workflow.

- Dataflow to Computation time Ratio (DCR) is the ratio of the average dataflow time to average computation time in a workflow. The dataflow time in a workflow is computed using (11).

$$
\text { dataflow time }=D C R * \text { computation time }
$$

when DCR $\leq 1$, computation - intensive workflows can be generated while DCR $\geq 1$ workflows are data-intensive workflows.

- Shape parameter $(\alpha)$ determines the structure, i.e., the height and width of the workflow. The height of the workflow, i.e., the number of levels in a workflow is obtained by $\sqrt{n} / \alpha$ and the width of the workflow, i.e., the number of tasks at each level is $\sqrt{n} \times \alpha$. For $\alpha<1$ values, longer workflows with low parallelism are generated and when $\alpha>1$, shorter workflows with high parallelism can be generated.

- Heterogeneity factor $(\beta)$ determines the variation in the computation times of each the task on $m$ resources. Higher $\beta$ values cause much deviation in the computation times of the tasks while lesser $\beta$ values results in the trivial difference in the computation times of the tasks. The computation time of each task $t_{i}$ on the resource $r_{j}$ is denoted as $w_{i, j}$, where $1 \leq i \leq$ $n, 1 \leq j \leq m$, is randomly selected from the range computed using the equation (12)

$$
\overline{w_{i}} \times(1-\beta / 2) \leq w_{i, j} \leq \overline{w_{i}} \times(1+\beta / 2)
$$

where $\overline{w_{i}}$ is the average computation time of each task in the workflow.

To evaluate the effectiveness of the new makespan estimation model, two sets of randomly generated scientific workflows are used. The first set consists of computation-intensive workflows viz., LIGO and Epigenomics workflows which are generated with workflow size $(n)$ of $\{40,56,72,88\}$ and $\{20$, $32,64,106\}$ respectively.

As these workflows are computation-intensive applications, their DCR values cannot be more than one. Since the structure of the scientific workflows is known, the parameters such as workflow size $(n)$, DCR, heterogeneity factor $(\beta)$ and average computation time of the workflow $\left(\overline{w_{i}}\right)$ are essential for generating the scientific workflows. The characteristics of LIGO and Epigenomics workflow set for the experimentations are depicted in Table 2.

Another set of data-intensive workflows namely Cybershake and Montage with workflow sizes (n) $\{20,36,68,132\}$ and $\{20,38,59,98\}$ respectively, are generated. Since these workflows are data-intensive applications, their DCR values cannot be less than one and the parameters are specified in Table 3. The combination of the mentioned parameter values generated 2000 workflows for each scientific workflow. The proposed BCCT and WCCT values for varied resource set are computed for LIGO, Epigenomics, Cybershake and Montage workflows. The BCCT and WCCT are evaluated by considering the best and the worst of the three makespans generated by the HEFT, PETS, and MSL scheduling algorithms.

Table 2. Characteristics of LIGO and Epigenomics workflows

\begin{tabular}{|c|c|l|l|l|l|}
\hline Parameter & \multicolumn{5}{|c|}{ Values } \\
\hline DCR & 0.1 & 0.25 & 0.5 & 0.75 & 1.0 \\
\hline $\boldsymbol{\beta}$ & 0.1 & 0.25 & 0.5 & 0.75 & 1 \\
\hline$\overline{\boldsymbol{w}_{\boldsymbol{i}}}$ & 100 & 150 & 200 & 250 & \\
\hline $\boldsymbol{m}$ & 4 & 8 & 12 & 16 & 20 \\
\hline
\end{tabular}

Table 3. Characteristics of Cybershake and Montage workflows

\begin{tabular}{|c|c|c|c|c|c|}
\hline \multirow{2}{*}{ Parameter } & \multicolumn{5}{|c|}{ Values } \\
\hline DCR & 0.1 & 0.25 & 0.5 & 0.75 & 1.0 \\
\hline $\boldsymbol{\beta}$ & 0.1 & 0.25 & 0.5 & 0.75 & 1 \\
\hline$\overline{\boldsymbol{w}_{\boldsymbol{i}}}$ & 100 & 150 & 200 & 250 & \\
\hline $\boldsymbol{m}$ & 4 & 8 & 12 & 16 & 20 \\
\hline
\end{tabular}

The experimental results are presented in Table 4, 5, 6, and 7 respectively. Each row in these tables represents the average data from 20 workflows obtained with the combination of different $\beta$ and $\overline{w_{i}}$ values for each DCR value.

The validation of the new makespan estimation model is performed by comparing BCCT and WCCT values computed for each workflow with the actual makespan of the workflow. The error $\xi$ between WCCT and the actual makespan is

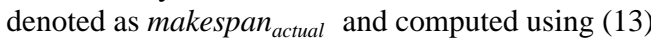

$$
\xi=\left|\frac{\text { makespan }_{\text {actual }}-\text { WCCT }}{\text { makespan }_{\text {actual }}}\right|
$$

From the Table.4, it can be observed that for the computationintensive LIGO workflows the error percent increased as DER value increased when the workflow size and the number of resources are same. Moreover, error enhanced as the workflow size increased for the same number of resources $(\mathrm{m})$ and DER values. The error in percent for the workflow size 40 is noted to be 7.65 , for 56 tasks it is 8.53 , for 72 tasks it is 10.64 and for 88 tasks the error 13.93 percent. An error is observed to be below 10 percent for 70 percent of the cases and overall it is 10.18 percent.

Moreover, the error varied for different number of resources $(m)$. For $m=\{4,8,12,16,20\}$ and the workflow size 40, the error percent is $\{8.2,7.9,7.2,7.4,7.5\}$, while for workflow size 56 , it is $\{7.8,13.0,8.0,6.8,7.1\}$, for workflow size 72 it is 
$\{7.0,16.6,14.8,8.1,6.8\}$, and for workflow size 88 it is $\{6.6,19.1,20.4,15.3,8.3\}$ respectively. For the same workflow size, the error is observed to vary for different number of resources. The error percent is noted to be high when the workflows attain optimal makespan. This implies that optimal schedules are attained when the number of resources provisioned are sufficient to execute the workflow.

Similar trends in the error values can also be observed for the computation-intensive Epigenomics workflow shown in Table 5. The error values are observed to be increasing with the rise

in the DER values. The error percent for workflow size $\{20$, $32,64,106\}$ is $\{10.89,11.33,8.81,10.61\}$ respectively. For various $m$ values i.e., $\{4,8,12,1620\}$, the error percent for workflow size 20 is $\{9.4,10.9,11.5,11.3,11.4\}$, for workflow size 32 it is $\{8.94,10.5,12.4,12.6,12.2\}$, for workflow size 64 it is $\{4.5,10.5,8.8,9.8,10.4\}$ and for 106 tasks it is $\{1.7,12.6$, $16.1,13.3,9.3$ \} respectively. Overall, the error value is noted to be 10.41 .

For a data-intensive Cybershake workflow presented in the Table 6, the error in percent for 20 tasks is 13.64 , for 36 tasks it is 13.92 , for 68 tasks it is 29.6 and for 106 tasks it is 47.2 . An overall error is noted to be 26 percent. For various $m$ values $\{4,8,12,16,20\}$, the error in percent for workflow size 20 is $\{17.5,12.4,12.8,12.8,12.7\}$, for workflow size 36 it is $\{17.7,18.8,14.5,9.4,9.2\}$, for 68 tasks it is $\{21.8,33.1,34.1,31.2,26.6\}$ and for 132 tasks it is $\{22.3,43.4,53.5,58.0,58.9\}$ respectively.

Table 4. Performance of the proposed BCCT and WCCT on LIGO Workflow set

\begin{tabular}{|c|c|c|c|c|c|c|c|c|c|c|c|c|c|c|c|c|c|c|c|c|c|c|}
\hline \multirow[b]{2}{*}{$n$} & \multirow[b]{2}{*}{ DER } & \multicolumn{4}{|c|}{$m=4$} & \multicolumn{4}{|c|}{$m=8$} & \multicolumn{4}{|c|}{$m=12$} & \multicolumn{4}{|c|}{$m=16$} & \multicolumn{4}{|c|}{$m=20$} & \multirow{2}{*}{$\begin{array}{l}\text { No. of } \\
\text { Work } \\
\text { flows }\end{array}$} \\
\hline & & BCT & \begin{tabular}{|l} 
Make \\
span
\end{tabular} & WCT & $\begin{array}{c}\text { Error } \\
(\%)\end{array}$ & BCT & $\begin{array}{c}\text { Make } \\
\text { span }\end{array}$ & WCT & $\begin{array}{c}\text { Error } \\
(\%)\end{array}$ & BCT & $\begin{array}{c}\text { Make } \\
\text { span }\end{array}$ & V & $\begin{array}{c}\text { Error } \\
(\%)\end{array}$ & $\mathbf{T}$ & $\begin{array}{c}\text { Make } \\
\text { span }\end{array}$ & WCT & $\begin{array}{c}\text { Error } \\
(\%)\end{array}$ & BC & $\begin{array}{c}\text { Make } \\
\text { span }\end{array}$ & VCT & $\begin{array}{c}\text { Error } \\
(\%)\end{array}$ & \\
\hline 40 & 0.1 & 1853 & 2368 & 2538 & 7.2 & 258 & 1813 & 948 & 7.4 & 100 & 1729 & 1844 & 6.6 & 1077 & 1694 & 1816 & 7.1 & 074 & 1701 & 822 & 7.1 & 20 \\
\hline 40 & 25 & 356 & 2441 & 630 & .7 & 52 & 928 & 75 & 7.6 & 00 & 1814 & 940 & 6.9 & 074 & 811 & 40 & 71 & 566 & 772 & 907 & 7.6 & 20 \\
\hline 40 & 0.5 & 1849 & 2476 & 2686 & 8.5 & 1244 & 1996 & 2152 & 7.8 & 1091 & 1875 & 2017 & 7.5 & 1082 & 1871 & 11 & 7.5 & 1073 & 877 & 016 & 7.4 & 20 \\
\hline 40 & 0.75 & 1848 & 2501 & 2728 & 9.1 & 1260 & 2024 & 2196 & 8.4 & 1100 & 1969 & 2115 & 7.4 & 1075 & 1929 & 2075 & 7.6 & 1069 & 1939 & 2089 & 7.7 & 20 \\
\hline 40 & 1 & 1823 & 2533 & 2754 & 8.8 & 1248 & 2059 & 2228 & 8.2 & 1095 & 1970 & 2116 & 7.4 & 1082 & 1967 & 2115 & 7.5 & 1072 & 1883 & 2028 & 7.7 & 20 \\
\hline 56 & 0.1 & 2395 & 2989 & 3188 & 6.7 & 1759 & 2039 & 300 & 12.8 & 1222 & 1825 & 1964 & 7.6 & 1080 & 1736 & 1850 & 6.5 & 1075 & 1722 & 1847 & 7.2 & 20 \\
\hline 56 & 0.25 & 2359 & 3057 & 3272 & 7.0 & 1772 & 2125 & 2397 & 12.8 & \begin{tabular}{|l|}
1226 \\
\end{tabular} & 1902 & 2057 & 8.1 & \begin{tabular}{|l|}
1079 \\
\end{tabular} & 1830 & 1961 & 7.1 & \begin{tabular}{|l|}
1068 \\
\end{tabular} & 1815 & 1955 & 7.7 & 20 \\
\hline 56 & & 362 & 3073 & 319 & 8.0 & 766 & 6 & 93 & 2.5 & 1224 & 2013 & 2170 & 7. & 1084 & 947 & 073 & 6.5 & 75 & 961 & 086 & 6.3 & 20 \\
\hline 56 & 0.75 & 52 & 3077 & 340 & 8 & 777 & 6 & 27 & 3.5 & 1230 & 2061 & 235 & 8.4 & 1078 & 2014 & 151 & 6.8 & 55 & 995 & 141 & 7.3 & 20 \\
\hline 56 & - & 345 & 3096 & 3360 & 8.5 & 1767 & 2259 & 562 & 13.4 & 1219 & 2087 & 2253 & 7.9 & 1080 & 2021 & 162 & 6.9 & 1070 & 1940 & 2072 & 6.8 & 20 \\
\hline 72 & 0.1 & 2898 & 3643 & 3856 & 5.9 & 2275 & 2298 & 2665 & 16 & 1749 & 1940 & 2229 & 14.9 & 1207 & 1816 & 1954 & 7.6 & 1078 & 1769 & 1880 & 6.3 & 20 \\
\hline 72 & 0.25 & 2881 & 3655 & 3903 & 6.8 & 2291 & 2381 & 2767 & 16.2 & 1753 & 2032 & 2324 & 14.4 & 1213 & 1921 & 2077 & 8.1 & 1070 & 1863 & 1997 & 7.1 & 20 \\
\hline 72 & 0.5 & 2891 & 3695 & 3968 & 7.4 & 2287 & 2427 & 2836 & 16.9 & 1731 & 2118 & 2424 & 14.5 & 1211 & 1995 & 2159 & 8.2 & 1072 & 1958 & 2088 & 6.6 & 20 \\
\hline 72 & 0.75 & 2879 & 3770 & 4035 & 7.0 & 2305 & 2470 & 2879 & 16.6 & 1732 & 2153 & 2472 & 14.8 & 1208 & 2078 & 2245 & 8.0 & 1066 & 2008 & 2150 & 7.0 & 20 \\
\hline 72 & 1 & 72 & 3731 & 40 & & 37 & 2476 & 33 & 2 & \begin{tabular}{|l|}
1736 \\
\end{tabular} & 0 & 2500 & 15.4 & 1208 & 081 & 2 & 8.4 & 69 & 34 & 17 & 6.7 & 20 \\
\hline 88 & 0. & 3406 & 4292 & 4521 & .3 & 2804 & 2588 & 3062 & 18.3 & 2254 & 2087 & 2518 & 20.6 & 1732 & 1898 & 2201 & 15.9 & 1201 & 1809 & 1955 & 8.1 & 20 \\
\hline 88 & 0.25 & 3398 & 4258 & 541 & 6.7 & 2809 & 2657 & 3154 & 18.7 & 2262 & 2177 & 2617 & 20.2 & 1726 & 2027 & 2328 & 14.9 & 1205 & 1923 & 2084 & 8.3 & 20 \\
\hline 88 & 0.5 & 3409 & 4364 & 4639 & 6.3 & 2798 & 2694 & 3215 & 19.3 & 2254 & 2264 & 2725 & 20.3 & 1727 & 2080 & 2400 & 15.4 & 1202 & 2016 & 2180 & 8.1 & 20 \\
\hline 88 & 0.75 & 3385 & 4333 & 4652 & 7.3 & 2799 & 2718 & 3253 & 19.7 & 2259 & 2288 & 2752 & 20.3 & 1728 & 2134 & 2456 & 15.0 & 1204 & 2084 & 2247 & 7.8 & 20 \\
\hline 88 & 1 & 3398 & 4362 & 4676 & 7.2 & 2809 & 2750 & 3290 & 19.6 & 2263 & 2301 & 2772 & 20.5 & 1729 & 2176 & 2509 & 15.3 & 1206 & 1996 & 2173 & 8.8 & 20 \\
\hline
\end{tabular}

Table 5. Performance of the proposed BCCT and WCCT on Epigenomics Workflow set

\begin{tabular}{|c|c|c|c|c|c|c|c|c|c|c|c|c|c|c|c|c|c|c|c|c|c|c|}
\hline \multirow[b]{2}{*}{$n$} & \multirow[b]{2}{*}{ DER } & \multicolumn{4}{|c|}{$m=4$} & \multicolumn{4}{|c|}{$m=8$} & \multicolumn{4}{|c|}{$m=12$} & \multicolumn{4}{|c|}{$m=16$} & \multicolumn{4}{|c|}{$m=20$} & \multirow{2}{*}{$\begin{array}{l}\text { No. of } \\
\text { Work } \\
\text { flows } \\
\end{array}$} \\
\hline & & CT & $\begin{array}{l}\text { Make } \\
\text { span }\end{array}$ & VCT & \begin{tabular}{|c|} 
Error \\
$(\%)$
\end{tabular} & BCT & $\begin{array}{l}\text { Make } \\
\text { span }\end{array}$ & WCT & $\begin{array}{c}\text { Error } \\
(\%)\end{array}$ & BCT & $\begin{array}{r}\text { Make } \\
\text { span }\end{array}$ & CT & $\begin{array}{c}\text { Error } \\
(\%)\end{array}$ & BCT & $\begin{array}{l}\text { Make } \\
\text { span }\end{array}$ & WCT & $\begin{array}{c}\text { Error } \\
(\%)\end{array}$ & BCT & $\begin{array}{l}\text { Make } \\
\text { span }\end{array}$ & Wo & $\begin{array}{c}\text { Error } \\
(\%)\end{array}$ & \\
\hline 20 & 0.1 & 91 & 1566 & 1698 & 84 & 11 & 1510 & 1654 & & 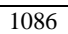 & 1464 & 1 & 10.0 & 1082 & & 1620 & 00 & 2 & & & & 20 \\
\hline 20 & & & 1696 & & & & & & & & & & & & & & & & & & & \\
\hline 20 & - & 96 & 1679 & & .0 & & 617 & & 1.0 & 92 & 536 & & & 75 & 560 & & & & 583 & & 1.3 & 0 \\
\hline 20 & 75 & & 1734 & 1908 & (2) & 123 & 1680 & & 11.5 & $j 96$ & 1613 & & 12 & 074 & 1574 & & & 1064 & 614 & & & 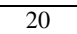 \\
\hline 20 & 1 & 93 & 1735 & 1914 & 10.3 & \begin{tabular}{|l|}
1117 \\
\end{tabular} & 1670 & 1862 & 11.5 & 091 & 1641 & 1836 & 11.9 & \begin{tabular}{|l|}
1039 \\
\end{tabular} & 1530 & 701 & 111.2 & \begin{tabular}{|l|}
1065 \\
\end{tabular} & 1585 & 1771 & 11.7 & 0 \\
\hline 32 & 0.1 & 759 & 3555 & 3759 & 7.2 & \begin{tabular}{|l|}
1923 \\
\end{tabular} & 2471 & 2687 & 8.7 & 1372 & 1837 & 2041 & 11.1 & \begin{tabular}{|l|}
1340 \\
\end{tabular} & 1817 & 2024 & 11.4 & 1344 & 1839 & 2041 & 11.0 & 20 \\
\hline 32 & 25 & 07 & 2329 & 2618 & 12.4 & \begin{tabular}{|l|}
1917 \\
\end{tabular} & 2503 & 2756 & 10.1 & 368 & 1911 & 2127 & 11 & 1345 & 1870 & 107 & 12.7 & 1333 & 1889 & 2112 & & 0 \\
\hline 32 & 0.5 & 33 & 2045 & 2271 & 11.0 & \begin{tabular}{|l|}
1902 \\
\end{tabular} & 2556 & 2827 & 10.6 & 367 & 1948 & 2197 & 12 & \begin{tabular}{|l|}
1348 \\
\end{tabular} & 1938 & 190 & & 1336 & 1934 & 5 & 2.5 & 20 \\
\hline 32 & & & 1951 & & & & 2574 & & & & 1983 & 2251 & & & 2018 & 3 & & 34 & 1992 & & & \\
\hline 32 & 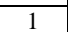 & & 18 & & & & & & & & & & & & 1996 & & & & & & & \\
\hline 64 & & & 3728 & & & & 2471 & & & & 2060 & & & & 1941 & & & & 1929 & & 9.4 & \\
\hline 64 & 25 & 39 & 3714 & 3869 & - & 7 & 2503 & \begin{tabular}{|l}
2756 \\
\end{tabular} & 10.1 & 374 & 2169 & 2346 & 8.2 & \begin{tabular}{|l|}
1351 \\
\end{tabular} & 2074 & 270 & 9.5 & 1342 & 2038 & 246 & 10.2 & 0 \\
\hline 64 & 0.5 & 37 & 3793 & 3967 & 4.6 & \begin{tabular}{|l|}
1902 \\
\end{tabular} & 2556 & 2827 & 10.6 & 365 & 2292 & 2481 & 8.3 & \begin{tabular}{|l}
1345 \\
\end{tabular} & 2162 & 371 & 9.6 & \begin{tabular}{|l|}
1341 \\
\end{tabular} & 2156 & 372 & 10.0 & 20 \\
\hline 64 & 0.75 & 18 & 3731 & 3938 & 5.6 & \begin{tabular}{|l|}
1912 \\
\end{tabular} & 2574 & 2864 & 11.3 & 367 & 2307 & 2520 & 9.2 & 1347 & 2237 & 2456 & 9.8 & 1341 & 2135 & 2380 & 11.4 & 20 \\
\hline 64 & 1 & 26 & 3779 & 3985 & 5.4 & \begin{tabular}{|l|}
1934 \\
\end{tabular} & 2587 & 2892 & 11.8 & 360 & 2237 & 2473 & 10.6 & 1348 & 2202 & 2440 & 10.8 & 1336 & 2101 & 2331 & 11.0 & 20 \\
\hline 106 & 0.1 & 37 & 5321 & 5360 & 207 & 32 & 3081 & 3430 & & 38 & 2412 & 2750 & $\overline{14}$ & 1860 & 2064 & 2333 & 13.0 & 1328 & 1873 & 5 & 8.7 & 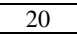 \\
\hline 106 & 0.25 & 40 & 5327 & 54 & & & 3120 & 34 & & & 2366 & 2750 & 16 & \begin{tabular}{|l|}
1860 \\
\end{tabular} & 2156 & 2448 & 13.5 & 1331 & 2018 & & 8.5 & \\
\hline 106 & & & 5349 & 544 & & 1 & 3141 & & & 2391 & 2400 & 2808 & 17. & \begin{tabular}{|l|}
1887 \\
\end{tabular} & 2022 & 216 & & 1335 & 2059 & 2256 & 9.5 & \\
\hline 106 & 0.75 & ד & 5374 & 548 & & 921 & 3178 & 3590 & & 2387 & 2486 & 2896 & 16. & 1933 & 2294 & 638 & 15.0 & 1334 & 2051 & 2263 & 10.3 & \\
\hline 106 & 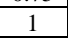 & 3503 & 5299 & 5434 & 2.5 & \begin{tabular}{|l|}
2937 \\
\end{tabular} & 3137 & \begin{tabular}{|l}
3566 \\
\end{tabular} & 13.7 & 2390 & 2495 & 2917 & 16.9 & \begin{tabular}{|l|}
1851 \\
\end{tabular} & 2203 & 2539 & 15.3 & 1372 & 2056 & 2254 & 9.6 & 20 \\
\hline
\end{tabular}


Table 6. Performance of the proposed BCCT and WCCT on Cybershake Workflow set

\begin{tabular}{|c|c|c|c|c|c|c|c|c|c|c|c|c|c|c|c|c|c|c|c|c|c|c|}
\hline \multirow[b]{2}{*}{$n$} & \multirow[b]{2}{*}{ DER } & \multicolumn{4}{|c|}{$m=4$} & \multicolumn{4}{|c|}{$m=8$} & \multicolumn{4}{|c|}{$m=12$} & \multicolumn{4}{|c|}{$m=16$} & \multicolumn{4}{|c|}{$m=20$} & \multirow{2}{*}{$\begin{array}{l}\text { No. of } \\
\text { Work } \\
\text { flows }\end{array}$} \\
\hline & & BCT & \begin{tabular}{|l} 
Make \\
span
\end{tabular} & VCT & \begin{tabular}{|c|} 
Error \\
$(\%)$
\end{tabular} & BCT & \begin{tabular}{|l|} 
Make \\
span \\
\end{tabular} & WCT & \begin{tabular}{|c|} 
Error \\
$(\%)$
\end{tabular} & BCT & $\begin{array}{r}\text { Make } \\
\text { span }\end{array}$ & WCT & \begin{tabular}{|c} 
Error \\
$(\%)$
\end{tabular} & BCT & \begin{tabular}{|l}
$\begin{array}{l}\text { Make } \\
\text { span }\end{array}$ \\
\end{tabular} & WCT & $\begin{array}{c}\text { Error } \\
(\%)\end{array}$ & BCT & \begin{tabular}{|c|} 
Make \\
span \\
\end{tabular} & WCT & \begin{tabular}{|c|} 
Error \\
$(\%)$
\end{tabular} & \\
\hline 20 & 1 & 173 & 292 & 342 & \begin{tabular}{|l|}
17.0 \\
\end{tabular} & 165 & 274 & 300 & 9.6 & 162 & 269 & 296 & \begin{tabular}{|c|}
10.2 \\
\end{tabular} & 160 & 269 & 297 & 10.4 & 158 & 270 & 297 & 10.0 & 20 \\
\hline 20 & 2 & & 4 & 6 & & 55 & 357 & & 10.0 & & 358 & 394 & & 160 & 357 & 94 & & 161 & 354 & 2 & 0.8 & 0 \\
\hline 20 & 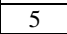 & & & & & & & & & 162 & & & & & 593 & 665 & & & 607 & & & 20 \\
\hline 20 & 7 & 73 & 729 & 9 & .9 & 56 & 123 & 824 & .0 & 163 & 728 & 830 & .9 & 161 & 711 & 814 & +.5 & 159 & 718 & 21 & 4.4 & 20 \\
\hline 20 & 10 & 2 & 896 & 1072 & 19.7 & 164 & 892 & 1041 & 16.7 & 163 & 894 & 1043 & 16.8 & 160 & 904 & 1053 & 16.6 & 160 & 765 & 894 & 16.8 & 20 \\
\hline 36 & 1 & 77 & 439 & 516 & 17.6 & 166 & 316 & 390 & 23.5 & 164 & 284 & 337 & 18.8 & 160 & 285 & 310 & 8.7 & 160 & 278 & 303 & 9.0 & 20 \\
\hline 36 & 2 & 74 & 512 & 603 & \begin{tabular}{|l|l|}
17.8 \\
\end{tabular} & 170 & 404 & 488 & 20.9 & 165 & 387 & 447 & 15.6 & 160 & 384 & 417 & 8.4 & 159 & 385 & 417 & 8.5 & 20 \\
\hline 36 & 5 & 31 & 731 & 862 & 17.9 & 168 & 653 & 771 & 18.1 & 164 & 662 & 750 & 13.2 & 160 & 642 & 705 & 9.9 & 159 & 646 & 709 & 9.7 & 20 \\
\hline 36 & 7 & & 858 & 1017 & 18.6 & 165 & 829 & 966 & 16.5 & 162 & 812 & 925 & 13.9 & 160 & 813 & 896 & 10.2 & 159 & 822 & 903 & 9.9 & 20 \\
\hline 36 & 10 & & 1133 & 1321 & 16.6 & 169 & 1103 & 1266 & 4.8 & 163 & 1144 & 1273 & 11.3 & 160 & 1122 & 1229 & 9.5 & 160 & 960 & 1047 & 9.1 & 20 \\
\hline 68 & 1 & & 657 & 782 & 10.0 & 166 & 396 & 541 & 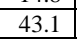 & 164 & 325 & 459 & 48 & 160 & 294 & 409 & 45 & 160 & 286 & 379 & 38.4 & 20 \\
\hline 68 & 2 & & 739 & 3 & & 170 & 483 & 638 & 37.6 & 165 & 415 & 556 & 40.1 & 160 & 389 & 511 & 37.0 & 159 & 382 & 482 & 30.8 & 20 \\
\hline 68 & 5 & & 929 & 1102 & 1.9 & 168 & 710 & 896 & 30.8 & 164 & 655 & 827 & 30.7 & 160 & 642 & 792 & 27.5 & 159 & 635 & 761 & 23.4 & 20 \\
\hline 68 & 7 & $1 / 4$ & 1068 & 1264 & 21.6 & 165 & 848 & 1057 & 29.0 & 162 & 814 & 1005 & 27.6 & 160 & 806 & 975 & 24.7 & 159 & 806 & 949 & 20.8 & 20 \\
\hline 68 & 10 & 178 & 1255 & 1489 & 21.9 & 169 & 1105 & 1340 & 25.1 & 163 & 1078 & 1293 & 23.5 & 160 & 1076 & 1270 & 21.2 & 160 & 926 & 1078 & 19.4 & 20 \\
\hline 132 & 1 & & 1171 & 1439 & 22.9 & 139 & 653 & 997 & 52.7 & 135 & 481 & 831 & 72.6 & 133 & 398 & 737 & 85.3 & 132 & 361 & 679 & 88.1 & 20 \\
\hline 132 & 2 & & 1266 & 1543 & 21.9 & 139 & 734 & 1090 & 48.5 & 135 & 568 & 929 & 63.6 & 134 & 492 & 840 & 70.6 & 133 & 453 & 779 & 72.1 & 20 \\
\hline 132 & 5 & & 1458 & 1780 & & 141 & 941 & 1337 & & 138 & 797 & 1196 & 50.0 & 135 & 734 & 1116 & 52.1 & 134 & 714 & 1072 & 50.1 & 20 \\
\hline 132 & 1 & & 1580 & 1931 & 22.2 & 136 & 1088 & 1511 & 38.9 & 138 & 962 & 1382 & 43.1 & 133 & 902 & 1304 & 44.1 & 132 & 882 & 1264 & 43.3 & 20 \\
\hline 132 & 10 & 146 & 1780 & 2178 & 22.3 & 137 & 1323 & 1782 & \begin{tabular}{|l|}
34.7 \\
\end{tabular} & 136 & 1208 & 1661 & 37.5 & 133 & 1165 & 1599 & 37.2 & 133 & 966 & 1361 & 41.0 & 20 \\
\hline
\end{tabular}

Table 7. Performance of the proposed BCCT and WCCT on Montage Workflow set

\begin{tabular}{|c|c|c|c|c|c|c|c|c|c|c|c|c|c|c|c|c|c|c|c|c|c|c|}
\hline \multirow[b]{2}{*}{$n$} & \multirow[b]{2}{*}{ DER } & \multicolumn{4}{|c|}{$m=4$} & \multicolumn{4}{|c|}{$m=8$} & \multicolumn{4}{|c|}{$m=12$} & \multicolumn{4}{|c|}{$m=16$} & \multicolumn{4}{|c|}{$m=20$} & \multirow{2}{*}{$\begin{array}{l}\text { No. of } \\
\text { Work } \\
\text { flows }\end{array}$} \\
\hline & & BCT & $\begin{array}{l}\text { Make } \\
\text { span }\end{array}$ & WCT & $\begin{array}{c}\text { Error } \\
(\%)\end{array}$ & BCT & \begin{tabular}{|l|} 
Make \\
span \\
\end{tabular} & WCT & $\begin{array}{c}\text { Error } \\
(\%)\end{array}$ & CT & $\begin{array}{r}\text { Make } \\
\text { span }\end{array}$ & WCT & $\begin{array}{c}\text { Error } \\
(\%)\end{array}$ & BCT & \begin{tabular}{|c|} 
Make \\
span
\end{tabular} & WCT & \begin{tabular}{|c} 
Error \\
$(\%)$
\end{tabular} & $\Gamma$ & \begin{tabular}{|c|}
$\begin{array}{c}\text { Make } \\
\text { span }\end{array}$ \\
\end{tabular} & T & $\begin{array}{c}\text { Error } \\
(\%)\end{array}$ & \\
\hline 20 & 1 & 22 & 474 & 537 & 13.2 & 303 & 468 & 31 & 13.3 & 299 & 480 & 537 & $\begin{array}{l}11.9 \\
\end{array}$ & 295 & 465 & 527 & 13.3 & 292 & 459 & 525 & 14.3 & 20 \\
\hline 20 & 2 & 27 & 624 & 18 & 5.2 & 308 & 618 & 721 & 6.6 & 298 & 609 & 712 & 5.8 & 95 & 607 & 712 & 7.4 & 292 & 612 & 715 & 9 & 20 \\
\hline 20 & 5 & 23 & 817 & 996 & 29.2 & 307 & 856 & 1024 & 32.8 & 299 & 857 & 1023 & 32.2 & 295 & 850 & 1016 & 32.4 & 293 & 852 & 1018 & 32.4 & 20 \\
\hline 20 & 7 & 327 & 870 & 1142 & 41.7 & 304 & 882 & \begin{tabular}{|l|}
1139 \\
\end{tabular} & 48.4 & 298 & 873 & 1130 & 48.9 & 296 & 892 & 1143 & 47.1 & 295 & 905 & 1156 & 46.4 & 20 \\
\hline 20 & 10 & 19 & 720 & 1005 & 79.0 & 307 & 726 & \begin{tabular}{|l|}
1007 \\
\end{tabular} & 96.5 & 300 & 727 & 1005 & 95.8 & 296 & 721 & 1000 & 96.8 & 293 & 673 & 900 & 84.5 & 20 \\
\hline 38 & 1 & 27 & 628 & 714 & 13.8 & 310 & 554 & 643 & 16.2 & 299 & 548 & 605 & 10.3 & 293 & 537 & 595 & 10.8 & 293 & 533 & 594 & 11.4 & 20 \\
\hline 38 & 2 & 77 & 779 & 22 & 3.4 & 302 & 751 & 894 & 19 & 298 & 754 & 863 & 14 & 295 & 747 & 861 & 15.3 & 292 & 741 & 853 & 5.1 & 20 \\
\hline 38 & 5 & 20 & 1135 & 1459 & 3.6 & 303 & 1138 & 1431 & 32.3 & 299 & 1156 & 1410 & 27 & 294 & 1139 & 1409 & 29.6 & 293 & 1129 & 1400 & 30.0 & 20 \\
\hline 38 & 7 & 22 & 1370 & 322 & 3.0 & 301 & 1374 & 1781 & 37.1 & 301 & 1377 & 1765 & 35.2 & 295 & 1328 & 1746 & 39.3 & 293 & 1367 & 1757 & 35.6 & 20 \\
\hline 38 & 10 & 20 & 1640 & 2218 & 39.2 & 303 & 1619 & 2185 & 48.6 & 298 & 1629 & 2165 & 45.7 & 294 & 1645 & 2171 & 44.3 & 294 & 1388 & 1829 & 44.1 & 20 \\
\hline 59 & 1 & 24 & 774 & 871 & 12.5 & 306 & 582 & 715 & 23.0 & 300 & 551 & 657 & 19.3 & 299 & 545 & 616 & 13.0 & 294 & 541 & 588 & 8.8 & 20 \\
\hline 59 & 2 & 25 & 909 & 1041 & 14.5 & 301 & 736 & 909 & 23.5 & 296 & 718 & 860 & 19.8 & 298 & 722 & 829 & 14.9 & 298 & 716 & 798 & 11.5 & 20 \\
\hline 59 & 5 & 35 & 1302 & 1540 & 18.2 & 306 & 1225 & 1508 & 23.1 & 299 & 1219 & 1468 & 20.4 & 295 & 1216 & 1431 & 17.7 & 292 & 1225 & 1414 & 15.5 & 20 \\
\hline 59 & 7 & 25 & 1621 & 1920 & 18.5 & 309 & 1560 & 1915 & 22.7 & 300 & 1558 & 1877 & 20.5 & 301 & 1558 & 1845 & 8.5 & 292 & 1558 & 1817 & 16.6 & 20 \\
\hline 59 & 10 & 1 & 2095 & 2481 & .4 & 304 & 2072 & 2531 & 2 & 300 & 2066 & 2492 & 20.6 & 297 & 2069 & 2462 & 19.0 & 297 & 1749 & 2049 & 17.1 & 20 \\
\hline 98 & 1 & 19 & 1097 & 1234 & 2.5 & 307 & 734 & 961 & 30.9 & 298 & 633 & 847 & 33.8 & 295 & 597 & 787 & 32.0 & 291 & 584 & 741 & 26.9 & 20 \\
\hline 98 & 2 & 21 & 1188 & 1392 & 17.2 & 305 & 895 & 1191 & 33.0 & 299 & 858 & 1131 & 31.8 & 296 & 855 & 1092 & 27.8 & 293 & 850 & 1055 & 24.1 & 20 \\
\hline 98 & 5 & 319 & 1615 & 2008 & 24.4 & 303 & 1539 & 2007 & 30.4 & 301 & 1531 & 1968 & 28.5 & 294 & 1533 & 1938 & 26.5 & 294 & 1512 & 1893 & 25.2 & 20 \\
\hline 98 & 7 & 318 & 1942 & 2449 & 26.1 & 304 & 1918 & \begin{tabular}{|l|}
2528 \\
\end{tabular} & 31.8 & 298 & 1931 & 2490 & 29.0 & 294 & 1922 & 2449 & 27.4 & 292 & \begin{tabular}{|l|}
1936 \\
\end{tabular} & 2428 & 25.4 & 20 \\
\hline 98 & 10 & 321 & 2406 & 3103 & 29.0 & 306 & 2432 & 3252 & 33.7 & 300 & 2366 & 3169 & 33.9 & 295 & 2358 & 3113 & 32.0 & 295 & 2008 & 2615 & 30.2 & 20 \\
\hline
\end{tabular}

For Montage workflow depicted in the Table 7, for lower workflow sizes the error percent is high and as the workflow size increased the error is observed to decline. It can be observed that error increased with DER values. The error for the workflow size 20 is observed to be 39.72 percent, it is 27.82 percent for 38 tasks, 17.98 percent for 59 tasks, and increased to 28 percent for 98 tasks. And overall error is 28 percent.

\section{CONCLUSION}

A new makespan estimation model is devised to estimate the bounds on the makespan of the workflows. In the available model's dataflows were either ignored or nominal, hence these models could not be extended for computation-intensive and data-intensive workflows. The proposed makespan estimation model is devised with minimal information considering the profile of the workflow as the makespan of the workflow highly depends on its profile. The proposed estimation model is devised with a complexity of $\mathrm{O}(n)$ and $\mathrm{O}(n+e)$ for computing BCCT and WCCT respectively. The present model is validated using the scientific workflows. The results of the experiments revealed that the proposed model could precisely estimate the makespan of the scientific workflows. The error for computation-intensive workflows namely LIGO and Epigenomics is observed to be 10.18 and 10.4 percent respectively and for data-intensive workflows namely Cybershake and Montage it is noted to be 26 percent and 28 percent respectively.

\section{REFERENCES}

[1] M.R., and D.S.Johnson, "Computers and intractability: A guide to the theory of NP-completeness," W.H.Freeman and Co., San Franisco, CA, 1979.

[2] H.Topcuoglu, S.Hariri, and M.Y.Wu, "Performance effective and low-complexity task scheduling for heterogeneous computing," IEEE Trans. Parallel Distributed Systems,vol.13 (3), pp.260-274, 2002.

[3] D.Sirisha, and G.Vijayakumari, "Minimal start time heuristics for scheduling workflows in heterogeneous processing systems, " Distributed Computing and Internet Technology, Springer Lecture Notes in Computer Science, vol.9581, pp.199-212, 2016.

[4] E.B.Fernandez, and B.Bussell, "Bounds on the number of resources and time for multiresource optimal schedules," IEEE Trans. Computers, vol.22(8), pp.745751,1973 . 
[5] K. Jain Kumar, and V. Rajaraman, "Lower and upper bounds on time for multiresource optimal schedules," IEEE Transactions on Parallel and Distributed Systems, vol.5(8), pp.879-886,1994.

[6] S. Bharathi, A. Chervenak, E. Deelman, G. Mehta, M.-H. $\mathrm{Su}$, and $\mathrm{K}$. Vahi, "Characterization of scientific workflows," $3^{\text {rd }}$ Workshop on Workflows in Support of Large-Scale Science, pp.1-10, Nov. 2008.

[7] E.Illavarasan, and P.Thambidurai, "Low complexity performance effective task scheduling algorithm for heterogeneous computing environments," Journal of Computer Science, vol.3(2), pp.94-103, 2007.

[8] I. Pietri, G. Juve, E. Deelman and R. Sakellariou, "A Performance model to estimate execution time of scientific workflows on the cloud," $9^{\text {th }}$ Workshop on Workflows in Support of Large-Scale Science, New Orleans, LA, pp. 11-19, 2014.
[9] Berriman G, Laity A, Good J, Jacob J, Katz D, Deelman E, Singh G, Su M, Prince T., "Montage: The architecture and scientific applications of a national virtual observatory service for computing astronomical image mosaics," Proceedings of Earth Sciences Technology Conference, 2006.

[10] Graves R, Jordan TH, Callaghan S, Deelman E, Field E, Juve G, Kesselman C, Maechling P, Mehta G, Milner K, "Cybershake: A physics-based seismic hazard model for southern california," Pure and Applied Geophysics, vol. 168(3-4), pp.367-381,2011.

[11] Abramovici A, Althouse WE, Drever RW, G“ursel Y, Kawamura S, Raab FJ, Shoemaker D, Sievers L, Spero RE, Thorne KS, "Ligo: The laser interferometer gravitational-wave observatory Science," vol. 256 (5055), pp.325-333,1992.

[12] USC Epigenome Center. http :// epigenome.usc.edu. Accessed : October 2015. 